\title{
Estrogen Replacement Reduces Hearing Threshold Shifts and Cochlear Hair Cell Loss After Acoustic Overexposure in Ovariectomized Rats
}

\author{
Min Tae Kim ${ }^{1, *}$ (i) $\cdot$ Jae-Hun Lee ${ }^{1,2,3, *}$ (i) $\cdot$ Nathaniel T. Carpena ${ }^{2}$ (i] $\cdot$ Min Young Lee ${ }^{1,2,3}$ (i) \\ Phil-Sang Chung ${ }^{1,2,3}$ (D) Jae Yun Jung ${ }^{1,2,3}$ (D) \\ ${ }^{1}$ Department of Otolaryngology-Head and Neck Surgery, Dankook University Hospital, Cheonan; ${ }^{2}$ Department of Otolaryngology-Head and \\ Neck Surgery and ${ }^{3}$ Beckman Laser Institute Korea, Dankook University College of Medicine, Cheonan, Korea
}

Objectives. The relationship of estrogen (the primary female sex hormone) with hearing function has been studied in both humans and animals. However, whether estrogen levels affect hearing remains uncertain. Therefore, in this study, we investigated changes in the vulnerability of hearing to acoustic overexposure in ovariectomized female rats.

Methods. Eighteen 8-week-old female Sprague-Dawley rats were separated into four groups as follows: sham ovariectomy (OP), OP only, and OP treated with low $(10 \mu \mathrm{g} / \mathrm{kg})$ or high doses $(100 \mu \mathrm{g} / \mathrm{kg})$ of estrogen. Rats in the estrogen replacement groups were given two intraperitoneal injections. Hearing thresholds were measured before noise exposure, and at 1 day and 2 weeks after exposure.

Results. The hearing thresholds of the sham OP and OP-only groups were not significantly different. However, both estrogen groups showed a lower threshold shift than the OP-only group. Histological immunostaining analyses showed that hair cell loss in the $32 \mathrm{kHz}$ region was more severe in the sham OP group than in the OP-only group. Furthermore, there was little or no hair cell loss in either estrogen replacement group and significantly more hair cell loss in the OP-only group.

Conclusion. These results suggest that estrogen replacement may reduce the vulnerability of hearing to noise exposure in menopausal women.

Keywords. Hearing Loss; Estrogen Replacement Therapy; Ovariectomy

\section{INTRODUCTION}

Traumatic noise exposure causes excessive glutamate release in the synaptic clefts between hair cells and afferent nerve endings, resulting in hair cell loss and a shift in the hearing threshold [1].

\footnotetext{
- Received October 8, 2019

Revised December 31, 2019

Accepted February 5, 2020

- Corresponding author: Jae Yun Jung

Department of Otolaryngology-Head and Neck Surgery, Dankook

University College of Medicine, 119 Dandae-ro, Dongnam-gu,

Cheonan 31116, Korea

Tel: +82-41-550-3973, Fax: +82-41-556-1090

E-mail: jjkingy2k@gmail.com

*The first two authors contributed equally to this study.
}

Therefore, prevention and treatment of cochlear end-organ damage after noise exposure is crucial for preserving good hearing. Estrogen, which is the primary female sex hormone, is produced in ovaries, testes, and the adrenal glands. The influence of estrogen on auditory function is incompletely understood. The effect of estrogen is modulated by estrogen receptors alpha and beta. Both receptor subtypes are found in the cochlea, and they may have separate functions in hearing [2]. Experimental and clinical studies have suggested that reduced estrogen levels can negatively affect hearing through changes in neuronal or bone metabolism in otic structures, changes in the level of blood flow in the cochlea, and changes in neuro-regulation $[3,4]$.

The relationship between cochlear damage and estrogen has been studied in both human and animal models. Previous stud-

Copyright (C) 2021 by Korean Society of Otorhinolaryngology-Head and Neck Surgery.

This is an open-access article distributed under the terms of the Creative Commons Attribution Non-Commercial License (https://creativecommons.org/licenses/by-nc/4.0)

which permits unrestricted non-commercial use, distribution, and reproduction in any medium, provided the original work is properly cited. 


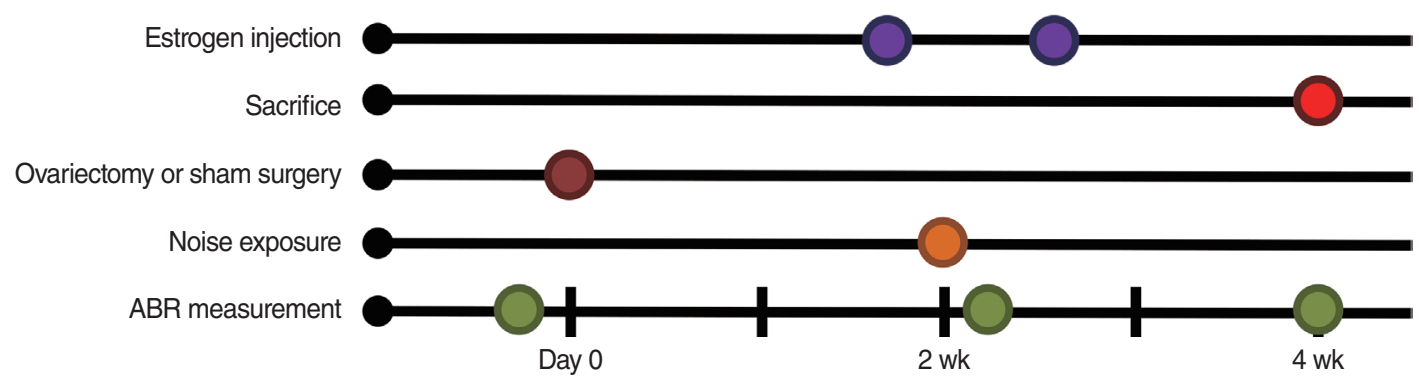

Fig. 1. Experimental schedule. Ovariectomy or sham surgery was performed 2 weeks before noise exposure (brown). Estrogen was injected 1 day before and 3 days after noise exposure (purple). Auditory brainstem response (ABR) was measured before and at 1 day and 2 weeks after noise exposure (green). The animals were sacrificed after the final ABR measurements (red).

ies have reported that estrogen protects neurons against degeneration $[5,6]$. An in vitro study using organ culture of the cochlea showed that estradiol, one of the forms of estrogen, protected the cochlea from damage caused by aminoglycosides by inhibiting the c-Jun N-terminal kinase pathway [7]. In addition, animals with ovaries had better hearing after acoustic trauma than animals with no ovaries [8]. However, neither of these results demonstrates that estrogen replacement therapy can preserve hearing after noise exposure. Furthermore, another animal study reported that estrogen treatment resulted in poorer hearing ability, as well as histological damage, as shown by vacuolization of the stria vascularis and inflammation [9]. In this regard, the timing, dose, and duration of hormone therapy are critical for its effects and more research is needed to address whether estrogen replacement therapy is beneficial for hearing vulnerability in post-menopausal woman. Therefore, in this study, we investigated the effect of estrogen replacement on hearing in ovariectomized female rats after traumatic noise exposure.

\section{MATERIAS AND METHODS}

All research procedures were approved by the Institutional Animal Care and Use Committee of Dankook University, Cheonan, Korea (No. DKU-17-028).

\section{Animals and research procedure}

Eighteen 8-week-old Sprague-Dawley rats were separated into four groups as follows: sham ovariectomy $(\mathrm{OP} ; \mathrm{n}=5)$, $\mathrm{OP}$ only

\section{H I}

- Ovariectomy did not change the vulnerability of hearing to noise exposure.

- Estrogen replacement therapy improved hearing threshold recovery after noise exposure.

- Estrogen administration reduced hair cell loss after noise exposure. $(\mathrm{n}=3)$, OP with low-dose estrogen replacement therapy (ET1; $\mathrm{n}=5$ ), and OP with high-dose estrogen replacement therapy (ET2; $\mathrm{n}=5$ ). All rats were anesthetized before auditory brainstem response (ABR) measurements were recorded and before surgery. After the baseline ABR measurements were recorded, surgery was performed on all rats. All animals were exposed to noise at 2 weeks after surgery and two different doses of estrogen therapy were administered to the treatment groups. To maintain estrogen levels during the noise stress and recovery periods, estrogen injections were given 1 day before and 3 days after noise exposure. ABR measurements were recorded again 1 day and 2 weeks after noise exposure (Fig. 1).

\section{Ovariectomy procedure}

Rats were anesthetized using $400 \mu \mathrm{L} / \mathrm{kg}$ intramuscular injections of zolazepam (Zoletil; Virbac, Carros, France) and xylazine (Rompun; Bayer AG, Leverkusen, Germany). The surgical procedures were performed in accordance with methods described previously $[10,11]$. Briefly, animals were placed in a prone position to facilitate access to both dorsolateral sides. Incisions (approximately $1.5-2 \mathrm{~cm}$ ) were made halfway between the middle of the back and the base of the tail and the ovarian fat pads were gently exposed using artery forceps. The ovaries were identified and gently removed (Supplementary Fig. 1). After both ovaries had been excised, the fat pads were repositioned inside the abdomen and muscles and skin were stitched up. After surgery, the animals were transferred to a heated pad for recovery. Sham OP group animals underwent a similar procedure but the ovaries were not removed.

\section{Noise exposure}

The rats were separated in different chambers to ensure they were equally exposed to noise. The chambers were placed in a sound reverberating acrylic noise box and the noise was introduced from a CP800Ti speaker in the ceiling (Beyma, Valencia, Spain). Narrow band noise (centered at $16 \mathrm{kHz}$ with $1 \mathrm{kHz}$ of band width) was generated using a type 1024 sine random generator (Bruel and Kjaer, Copenhagen, Denmark) and amplifier (Inter-M, Seoul, Korea). The rats were exposed to $120 \mathrm{~dB}$ sound 
pressure level (SPL) for 6 hours continuously. The noise level was monitored using a type 2250 frequency-specific sound level meter (Bruel and Kjaer).

\section{Estrogen replacement therapy}

$\beta$-estradiol powder (estradiol, E2758; Sigma-Aldrich, St. Louis, MO, USA) was dissolved in ethanol. The estradiol solution was diluted to the two working concentrations using distilled water. A total volume of $1 \mathrm{~mL}$ of estradiol was injected into the peritonea of rats in each of the estrogen replacement therapy groups $(10 \mu \mathrm{g} / \mathrm{kg}$ for ET1 and $100 \mu \mathrm{g} / \mathrm{kg}$ for ET2) 1 day before and 3 days after noise exposure. For OP-only and sham OP groups, only distilled water with same concentration of ethanol was injected.

\section{ABR measurements}

ABR thresholds were measured at baseline (before noise exposure) and 1 day and 2 weeks after noise exposure. Each animal was anesthetized and three subdermal needle electrodes were positioned as follows: one at the vertex (active) and one behind each pinna (reference/ground). Tone-burst stimuli were delivered through electrostatic speakers using an EC-1 insert tube (Tucker Davis Technologies, Alachua, FL, USA) and the response was measured at 8,16 , and $32 \mathrm{kHz}$ in $5 \mathrm{~dB}$ steps from 90 to $10 \mathrm{~dB}$ SPL using a System III evoked response signal-processing system (Tucker Davis Technologies). A total of 512 responses were amplified, filtered, and averaged to obtain the hearing thresholds. The stimulus intensity above the flat waveform was considered the threshold.

\section{Histological analysis}

After the final ABR measurements were recorded, the animals were sacrificed and the cochleae were carefully harvested. The cochleae were fixed in $10 \%$ neutral buffered formalin for 2 hours, decalcified in $0.1 \mathrm{M}$ EDTA (ethylenediaminetetraacetic acid) for 1 week, and gently microdissected as whole mount preparations. The samples were immunostained with antibodies against myosin VIIa (rabbit anti-myosin VIIa, 1:200; MilliporeSigma, Burlington, MA, USA) to identify hair cells and neurofilament-heavy (1:1,000, MilliporeSigma) to identify the auditory nerves. The appropriate secondary antibodies were coupled with the corresponding primary antibodies (anti-rabbit Alexa 568, 1:1,000 and anti-chicken Alexa 488, 1:1,000). After staining, the samples were mounted on slides using Vectashield H-1000 crystal mounting solution (Vector Laboratories, Burlingame, CA, USA).

\section{Confocal imaging and hair cell counting}

An FV-3000 confocal microscope (Olympus, Tokyo, Japan) with a $40 \times$ objective was used for imaging. The z-dimension was acquired at $1 \mu \mathrm{m}$ for each image stack. Images of the basilar membranes were obtained for 8,16 , and $32 \mathrm{kHz}$ [12]. Anti-myosin VIIa-positive inner (IHCs) and outer hair cells (OHCs) were count- ed within a square area $200 \mu \mathrm{m}$ in length.

\section{Statistical analysis}

All data were analyzed using IBM SPSS ver. 19.0 (IBM Corp., Armonk, NY, USA) or GraphPad Prism software (GraphPad Software, San Diego, CA, USA). Kolmogorov-Smirnov tests were used to determine data equivalence. Kruskal-Wallis tests and multivariate analysis of variance (MANOVA) were used to determine differences in hearing thresholds, threshold shifts, and hair cell loss among the groups. Univariate ANOVA and Tukey's test were used for post-hoc analyses.

\section{RESULTS}

To identify the traumatic effect of noise, the hearing thresholds in all groups were measured after noise exposure (Table 1, Fig. 2). At 1 day after noise exposure, the hearing thresholds in all groups significantly increased. The Kruskal-Wallis test was used to verify differences among the groups. However, there were no significant differences among the groups at any test frequency $(8 \mathrm{kHz}$ : $d f=3, P=0.132 ; 16 \mathrm{kHz}: d f=3, P=0.100 ; 32 \mathrm{kHz}: d f=3, P=0.254)$. Thus, we concluded that the noise exposure level used in this study could significantly increase the hearing threshold over a range of frequencies, without generating significant differences among subgroups.

To determine whether OP could affect the vulnerability of hearing to noise exposure, we compared the hearing thresholds of the OP-only and sham OP groups at two time points after noise exposure. At 1 day after noise exposure, the hearing thresholds of both groups had significantly increased and almost reached the maximum level at all test frequencies (Fig. 2B). At 2 weeks after noise exposure, the sham OP group had slightly lower hearing thresholds than the OP-only group at all test frequencies, but the differences were not statistically significant (Shapiro-Wilk test: parametric data, MANOVA; $8 \mathrm{kHz}: F=2.682, d f=3, P=0.941$; $16 \mathrm{kHz}: F=3.652, d f=1, P=0.854 ; 32 \mathrm{kHz}: F=5.974, d f=3, P=$

Table 1. Hearing thresholds in all groups at different time points

\begin{tabular}{ccccc}
\hline \multirow{2}{*}{ Frequency } & \multicolumn{4}{c}{ Group } \\
\cline { 2 - 5 } & Sham OP & OP only & ET1 & ET2 \\
\hline Baseline & \multicolumn{4}{c}{} \\
$8 \mathrm{kHz}$ & $18.3 \pm 2.9$ & $21.0 \pm 5.5$ & $20.0 \pm 6.1$ & $25.0 \pm 9.4$ \\
$16 \mathrm{kHz}$ & $20.0 \pm 10.0$ & $15.0 \pm 5.0$ & $19.0 \pm 7.4$ & $23.0 \pm 13.0$ \\
$32 \mathrm{kHz}$ & $16.7 \pm 7.6$ & $17.0 \pm 4.5$ & $19.0 \pm 7.4$ & $22.0 \pm 11.5$ \\
$24 \mathrm{Hours}$ after noise exposure & & & \\
$8 \mathrm{kHz}$ & $90.0 \pm 0.0$ & $87.0 \pm 4.5$ & $75.0 \pm 14.1$ & $85.0 \pm 8.7$ \\
$16 \mathrm{kHz}$ & $80.0 \pm 0.0$ & $74.0 \pm 4.2$ & $76.0 \pm 12.9$ & $76.0 \pm 4.2$ \\
$32 \mathrm{kHz}$ & $73.3 \pm 15.3$ & $79.0 \pm 6.5$ & $69.0 \pm 16.7$ & $74.0 \pm 4.2$ \\
\hline
\end{tabular}

Values are presented as mean \pm standard deviation.

OP, ovariectomy; ET1, low-dose estrogen replacement therapy; ET2, highdose estrogen replacement therapy. 
0.549) (Table 1, Fig. 3A). Therefore, the vulnerability of hearing to noise exposure was not changed by OP.

To investigate whether estrogen replacement therapy had a protective effect, changes in the hearing thresholds were evaluated at 2 weeks after noise exposure (Table 2). The hearing thresholds among the different groups varied significantly at 2 weeks after noise exposure. MANOVA showed significant differences in hearing thresholds among the groups at 8,16 , and $32 \mathrm{kHz}$ (MANOVA, $16 \mathrm{kHz}: F=3.652, d f=3, P=0.039 ; 32 \mathrm{kHz}: F=5.974$, $d f=3, P=0.008$ ). The Tukey post-hoc test showed significant differences between the OP-only and ET1 groups at $32 \mathrm{kHz}(P=$ 0.012), and between the OP-only and ET2 groups at $16 \mathrm{kHz}(P=$
$0.04)$ and $32 \mathrm{kHz}(P=0.02)$. The hearing thresholds of the sham $\mathrm{OP}$ and estrogen therapy groups were also compared, but no differences were found at any of the test frequencies at 2 weeks after noise exposure (sham OP vs. ET1: $8 \mathrm{kHz}, P=0.511 ; 16 \mathrm{kHz}$, $P=0.759 ; 32 \mathrm{kHz}, P=0.206$; sham OP vs. ET1: $8 \mathrm{kHz}, P=0.638$; $16 \mathrm{kHz}, P=0.561 ; 32 \mathrm{kHz}, P=0.298$ ) (Fig. $3 \mathrm{~A}$ ).

The magnitude of the threshold shifts was also compared among the test groups (Table 2, Fig. 3B). The threshold shifts between baseline and 2 weeks after noise exposure were calculated. The Shapiro-Wilk test showed that the results consisted of parametric data. MANOVA showed there were significant inter-group differences at $16 \mathrm{kHz}(F=3.652, d f=3, P=0.039)$ and $32 \mathrm{kHz}$
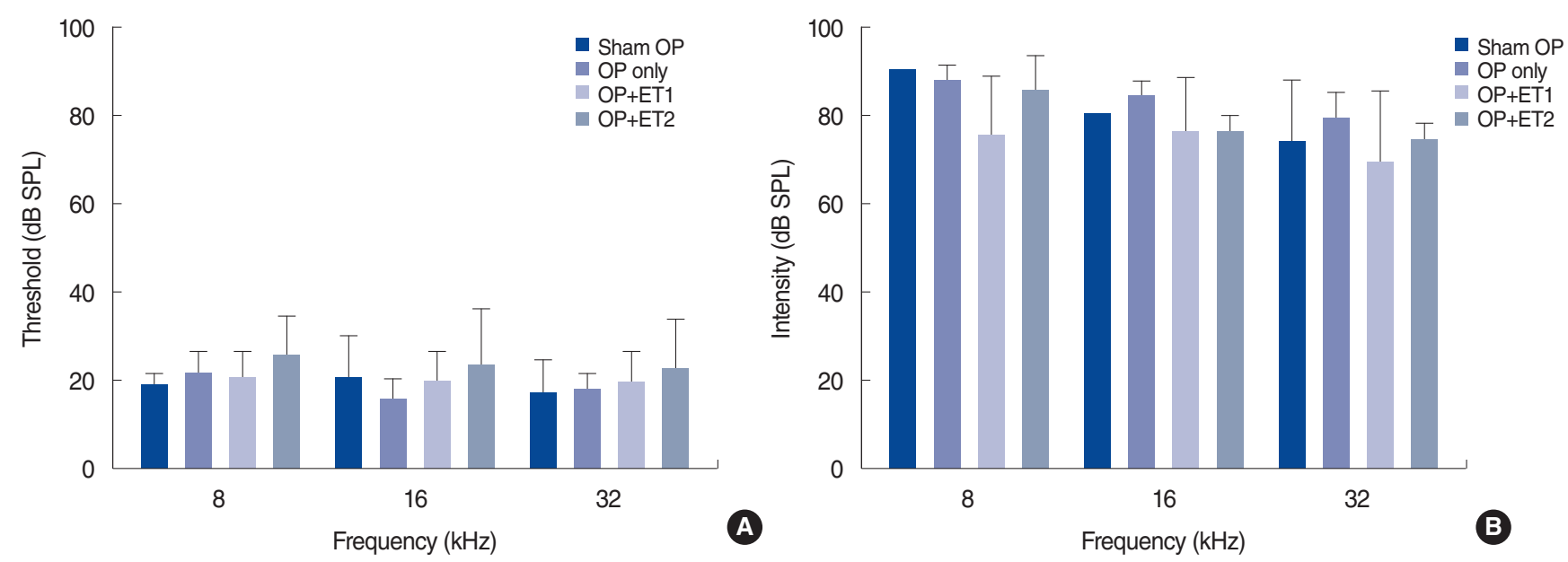

Fig. 2. Hearing thresholds increased after noise exposure in all groups. Hearing thresholds were measured before and 1 day after noise exposure. (A) Baseline hearing thresholds were normal in all experimental groups. (B) Hearing thresholds increased significantly 1 day after noise exposure in all groups and reached >80 dB SPL. Error bars indicate standard deviation. SPL, sound pressure level; OP, ovariectomy; ET1, low-dose estrogen replacement therapy; ET2, high-dose estrogen replacement therapy.
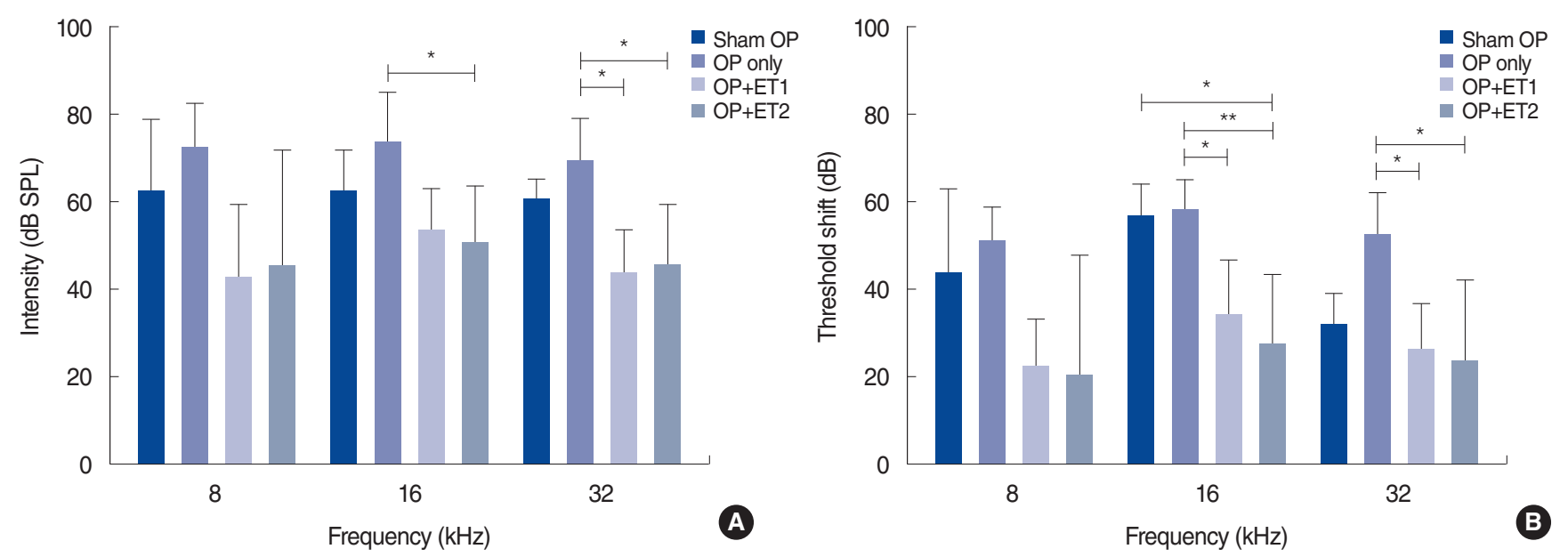

Fig. 3. Hearing thresholds were measured 2 weeks after noise exposure (NE), and threshold shifts from baseline were calculated. (A) Hearing thresholds among the different groups varied 2 weeks after NE. Significant differences were found between the OP-only and the estrogen replacement therapy groups at all test frequencies. (B) The threshold shifts between baseline and 2 weeks after NE varied among all groups. In addition, the threshold shift data between the OP-only and the estrogen replacement therapy groups differed significantly at all test frequencies. At $16 \mathrm{kHz}$, there was also a difference between the sham OP and ET2 groups. Error bars indicate standard deviation. SPL, sound pressure level; OP, ovariectomy; ET1, low-dose estrogen replacement therapy; ET2, high-dose estrogen replacement therapy. ${ }^{\star} P<0.05,{ }^{\star \star} P<0.01$. 
$(F=5.974, d f=3, P=0.008)$. The Tukey post-hoc test showed differences between the OP-only and ET1 groups at $16 \mathrm{kHz}(P=$

Table 2. Hearing thresholds and shifts in all groups at 2 weeks after noise exposure

\begin{tabular}{|c|c|c|c|c|}
\hline \multirow{2}{*}{ Frequency } & \multicolumn{4}{|c|}{ Group } \\
\hline & Sham OP & OP only & ET1 & ET2 \\
\hline \multicolumn{5}{|c|}{2 Weeks after noise exposure } \\
\hline $8 \mathrm{kHz}$ & $61.7 \pm 17.6$ & $72.0 \pm 10.4$ & $42.0 \pm 17.2$ & $45.0 \pm 26.7$ \\
\hline $16 \mathrm{kHz}$ & $61.7 \pm 10.4$ & $73.0 \pm 12.6$ & $53.0 \pm 10.4$ & $50.0 \pm 13.7$ \\
\hline $32 \mathrm{kHz}$ & $60.0 \pm 5.0$ & $69.0 \pm 10.3$ & $43.0 \pm 10.4$ & $45.0 \pm 14.6$ \\
\hline \multicolumn{5}{|c|}{ Threshold shift } \\
\hline $8 \mathrm{kHz}$ & $43.3 \pm 20.2$ & $51.0 \pm 8.2$ & $22.0 \pm 11.5$ & $20.0 \pm 28.3$ \\
\hline $16 \mathrm{kHz}$ & $56.7 \pm 7.6$ & $58.0 \pm 7.6$ & $34.0 \pm 12.9$ & $27.0 \pm 16.4$ \\
\hline $32 \mathrm{kHz}$ & $31.7 \pm 7.6$ & $52.0 \pm 10.4$ & $26.0 \pm 10.8$ & $23.0 \pm 19.2$ \\
\hline
\end{tabular}

Values are presented as mean \pm standard deviation. Scale indicates $\mathrm{dB}$ sound pressure level.

OP, ovariectomy; ET1, low-dose estrogen replacement therapy; ET2, highdose estrogen replacement therapy.
$0.035)$ and $32 \mathrm{kHz}(P=0.036)$. There were also significant differences between the OP-only and ET2 groups at $16 \mathrm{kHz}(P=0.006)$ and $32 \mathrm{kHz}(P=0.019)$. Furthermore, the threshold shift in the ET2 group was significantly smaller than that of the sham OP group at $16 \mathrm{kHz}(P=0.023)$. These results suggest that estrogen replacement therapy may enhance hearing threshold recovery or protect hearing against noise exposure. Moreover, at some frequencies, estrogen replacement therapy at high doses resulted in hearing thresholds that were better than those of non-ovariectomized animals.

To investigate the possibility of dose-dependent effects of estrogen replacement therapy, we compared the hearing thresholds and threshold shifts of the ET1 and ET2 groups. The mean hearing thresholds at 2 weeks after noise exposure and the threshold shifts were compared, and no significant differences were found at any test frequency (MANOVA: $8 \mathrm{kHz}, P=0.998 ; 16 \mathrm{kHz}, P=$ $0.850 ; 32 \mathrm{kHz}, P=1.000)$. These results indicate that estrogen replacement therapy did not show dose-dependent effects.

To verify histological changes in the organ of Corti after trau-

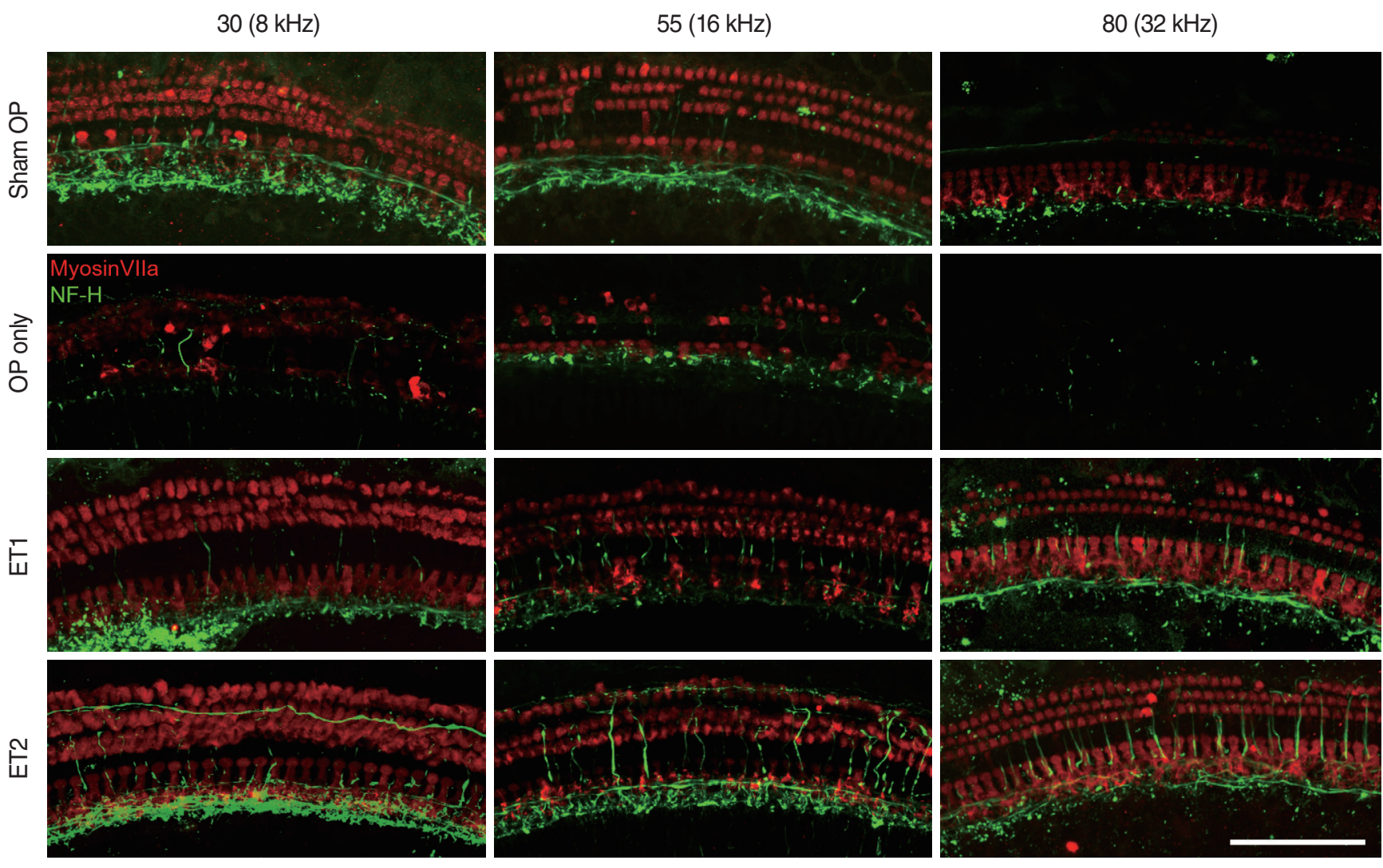

Fig. 4. Cochlear hair cells and the auditory nerve. Three parts of the basilar membrane (shown as the relative distance from the apex, \%), corresponding to different auditory brainstem response frequencies (8, 16, and $32 \mathrm{kHz}$ ), were immunostained for hair cells (myosin VIla, red) and nerve fibers (neurofilament-heavy, green). The sham OP group showed severe loss of both inner (IHCs) and outer hair cells (OHCs) at $32 \mathrm{kHz}$, slight loss of OHCs at $16 \mathrm{kHz}$, but intact hair cells in the $8 \mathrm{kHz}$ region of the basilar membrane. The OP-only group showed almost complete hair cell loss in the $32 \mathrm{kHz}$ region, severe $\mathrm{OHC}$ and slight $\mathrm{IHC}$ loss in the $16 \mathrm{kHz}$ region, and moderate $\mathrm{OHC}$ and $\mathrm{IHC}$ loss in the $16 \mathrm{kHz}$ region of the basilar membrane. For the estrogen replacement groups, both OHCs and IHCs remained nearly intact in every region except for a slight loss of OHCs in the ET1 group in the $32 \mathrm{kHz}$ region. Scale bar $=100 \mu \mathrm{m}$. OP, ovariectomy; ET1, low-dose estrogen replacement therapy; ET2, high-dose estrogen replacement therapy. 

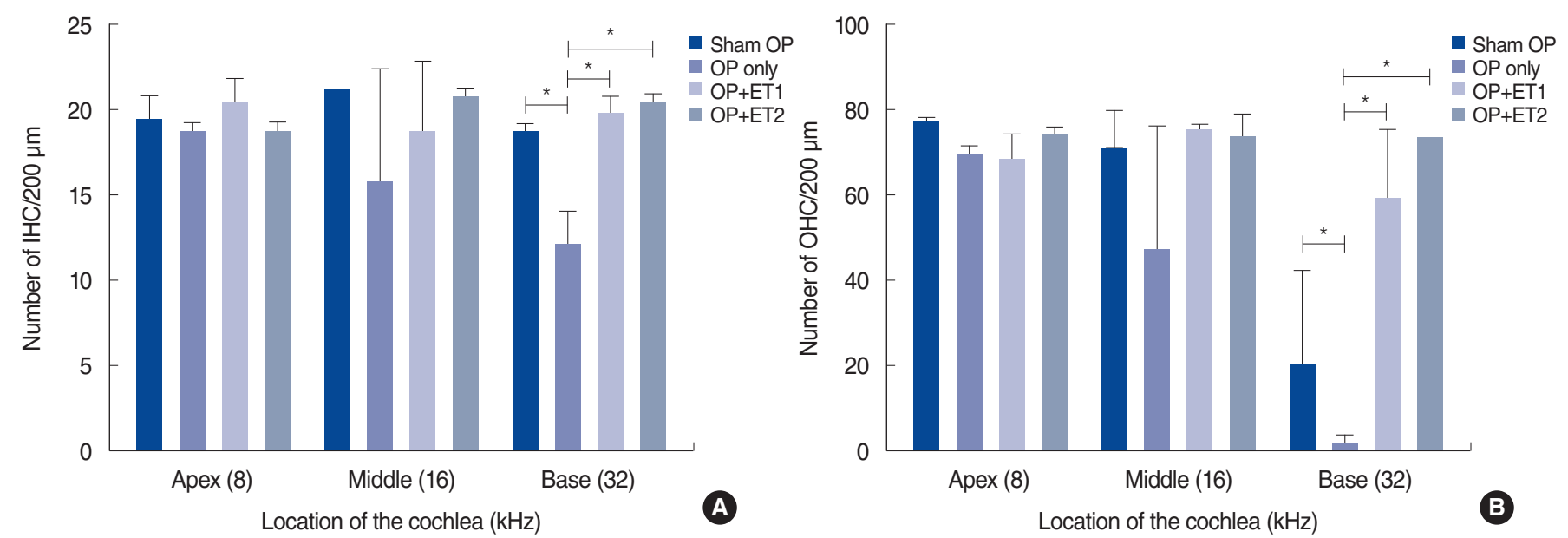

Fig. 5. Quantification of inner hair cells (IHCs; $A$ ) and outer hair cells $(\mathrm{OHCs}$; $\mathrm{B})$ in the cochlea. The number of $\mathrm{HCs}$ and OHCs within a square area $200 \mu \mathrm{m}$ in length were counted at three regions within the cochlea. There were no significant differences in the numbers of IHCs or OHCs among the groups. Error bars indicate standard deviation. OP, ovariectomy; ET1, low-dose estrogen replacement therapy; ET2, high-dose estrogen replacement therapy. ${ }^{*} P<0.05$.

matic noise exposure, the extracted cochlea samples were mounted and the hair cells and peripheral auditory nerves were visualized using immunofluorescence (Fig. 4). The sham OP group showed some $\mathrm{OHC}$ loss in the $16 \mathrm{kHz}$ region and severe $\mathrm{IHC}$ and $\mathrm{OHC}$ loss in the $32 \mathrm{kHz}$ region. The OP-only group showed more $\mathrm{OHC}$ loss than the sham OP group. The OP-only group showed slight IHC loss in the $8 \mathrm{kHz}$ region, severe OHC loss and mild IHC loss in the $16 \mathrm{kHz}$ region, and complete $\mathrm{OHC}$ and IHC loss in the $32 \mathrm{kHz}$ region. However, both estrogen replacement therapy groups had more intact hair cells than the other experimental groups. Both the ET1 and ET2 groups had intact hair cells in the 8 and $16 \mathrm{kHz}$ regions, and only slight $\mathrm{OHC}$ loss in the $32 \mathrm{kHz}$ region (Fig. 4).

The number of IHCs and OHCs were counted and compared among the groups (Fig. 5). The Shapiro-Wilk and Kruskal-Wallis $\left(x^{2}=8.953, P=0.03\right)$ tests showed significant differences in the number of IHCs among different groups. The Mann-Whitney $U$-test showed significant differences at $32 \mathrm{kHz}$ between the sham OP and OP-only groups $(P=0.046)$, the OP-only and ET1 groups $(P=0.046)$, and the OP-only and ET2 groups $(P=0.046)$.

The Shapiro-Wilk test also showed that the OHC data were non-parametric. The Kruskal-Wallis test showed significant differences at $32 \mathrm{kHz}\left(\chi^{2}=9.056, P=0.029\right)$. The Mann-Whitney $U$-test showed significant differences between the sham OP and OP-only groups $(P=0.046)$, the sham OP and ET2 groups $(P=$ $0.037)$, the OP-only and ET1 groups $(P=0.046)$, and the OP-only and ET2 groups $(P=0.034)$. These differences showed some similarities to the functional data (Fig. 4). From these results, we concluded that the histological changes partially matched the functional data, especially at $32 \mathrm{kHz}$. The differences between the histological and functional data at $16 \mathrm{kHz}$ may have been due to the small sample size.

\section{DISCUSSION}

\section{Overview of results}

In this study we used ABR thresholds to assess hearing in each animal. Significant threshold shifts in hearing function occurred after noise exposure, and were unaffected by the OP procedure. Different doses of estrogen in replacement therapy resulted in differences in hearing threshold recovery. The magnitude of the threshold shifts after noise exposure was significantly reduced at both estrogen concentrations. However, no dose-dependent effects of estrogen replacement therapy were observed. To investigate the histological basis of these observations, the number of IHCs and OHCs were counted in the regions responsible for different hearing frequencies. In contrast to the functional data, which revealed significant differences at all the frequencies tested, the histological results showed significant differences in the number of hair cells of both types only at the highest frequency. The estrogen replacement therapy groups had significantly more hair cells of both types than the OP group, and the OP group had fewer hair cells of both types than the sham OP control group. This differs from the functional data, which showed no differences between the OP and control groups. Possible reasons for differences between the functional and histological data are discussed below. Nevertheless, both the ABR and histological data suggest that estrogen replacement therapy, regardless of estrogen concentration, improved hearing and increased hair cell density compared to the OP-only animals, which reflected menopausal conditions.

The noise frequency used in this study was centered at 16 $\mathrm{kHz}$. However, the disruption of OHCs at $32 \mathrm{kHz}$ was more severe than at $16 \mathrm{kHz}$. This may have been due to differences in vulnerability between the apex and base of the cochlea. Different concentrations of glutathione generate differences in the 
susceptibility of hair and supporting cells to free radicals, resulting in different levels of damage in the apex and base when the cochlea is exposed to aminoglycosides or traumatic noise [13].

\section{Previous studies on the relationship between estrogen levels and hearing}

The relationship between hearing and estrogen levels has been studied extensively in humans and animal models. Previous studies in humans reported that ABR wave latencies were delayed and thresholds were higher in postmenopausal subjects and those with congenitally low levels of estrogen $[14,15]$. Previous studies in animals also showed that $A B R$ latencies were delayed after OP [8]. Naturally estrogen-deficient mice had prolonged ABR latencies compared to those of control mice [10]. These studies suggest that hearing thresholds and ABR peak latencies may be affected by internal estrogen levels. The effect of OP on hearing can be altered by external stimuli. Willot et al. [11] reported that ovariectomized mice showed smaller threshold shifts than control mice after noise exposure. In this study, we found no differences between sham OP and OP-only rats in hearing threshold or threshold shifts after noise exposure.

A limitation of this study is that we used an acute animal model to represent menopause. Furthermore, other organs, such as adipose tissue, may be able to compensate for estrogen depletion [12]. We did not analyze ABR wave latency in this study because the noise level used was sufficient to cause permanent threshold shifts, and the thresholds in both groups approached the level of deafness. Nevertheless, this limitation could have been responsible for the nonsignificant hearing threshold results.

\section{Effects of estrogen replacement therapy and possible mechanism}

In this study, both the ET1 and ET2 groups showed better functional and histological responses to noise exposure than the sham $\mathrm{OP}$ and OP-only groups. Several previous studies have shown that estrogen is protective against noise exposure and glutamate toxicity $[16,17]$. Estrogen can increase the production of the powerful antioxidants superoxide dismutase 2 and hydrogen sulfide, conferring protection against oxidative stress and decreasing the production of reactive oxygen species $[18,19]$. An estrogenstimulated increase in the production of brain-derived neurotrophic factor may also confer protective effects [16]. Although we did not investigate the pathways through which estrogen exerted its effects in this study, mechanisms similar to those reported in previous studies may have been involved.

Our functional and histological analyses found no differences between the ET1 and ET2 groups. This might have been because the low dose of estrogen $(10 \mu \mathrm{g} / \mathrm{kg})$ used in this study may already be high enough to produce the maximum effect possible. Coleman et al. [8] compared the effects of various doses of estrogen therapy in an ovariectomized rat model and found no dose-dependent effects on ABR. Another study demonstrated that different doses of estrogen did not increase the therapeutic effect if the lowest dose was already sufficient [20].

\section{Functional and histological mismatches}

We found two mismatches in the results of this study. First, hearing thresholds in all groups increased significantly, whereas improved histological results were observed at all the frequencies tested. Second, there was no significant hearing threshold difference between the sham OP and OP-only groups, whereas the histological results showed marked differences in hair cell survival at $32 \mathrm{kHz}$.

Loss of function in the remaining hair cells is a possible explanation for the first mismatch. The synapses between IHCs and nerve fibers are the most vulnerable part of the organ of Corti [21], and these synapses may be damaged even if all the hair cells remain intact. Furthermore, in cases of severe noise exposure, at least 2 months may be necessary for hair cell recovery [21]. Therefore, it is possible that further studies should record observations for more than 2 months to understand the mismatch between the histological and functional results described in this study.

Regarding the second mismatch, the level of noise may have been too high to investigate cochlear vulnerability due to OP. Because the thresholds in both the sham OP and OP-only groups approached the level of deafness, we surmise that the noise intensity might have offset any differences in hearing thresholds. Experimenting with lower levels of noise exposure may make it possible to identify differences in cochlear vulnerability between these groups.

\section{Limitations of this study}

Although the number of animals in each group was small, our histological results and images showed clear differences among the groups. Internal hormone levels can fluctuate after OP and estrogen replacement therapy and were not measured here. Nevertheless, the discrepancies between the functional and histological data may have occurred due to the factors described above. A larger sample size and comprehensive functional and histological analyses, such as peak 1 amplitude ABR, ribbon synapse analysis, and nerve structure studies are needed to confirm the findings presented in this study.

\section{CONFLICT OF INTEREST}

No potential conflict of interest relevant to this article was reported.

\section{ACKNOWLEDGMENTS}

This study was supported by the Ministry of Science, Informa- 
tion and Communications technology (ICT) and Future Planning grant funded by the Korean Government (NRF- 2017R1D1A1B03033219), and supported by Leading Foreign Research Institute Recruitment Program through the National Research Foundation of Korea (NRF) funded by the Ministry of Science and ICT (MSIT) (NRF- 2018K1A4A3A02060572).

\section{ORCID}

$\begin{array}{ll}\text { Min Tae Kim } & \text { https://orcid.org/0000-0003-3244-9024 } \\ \text { Jae-Hun Lee } & \text { https://orcid.org/0000-0003-2317-9958 } \\ \text { NathanielT. Carpena } & \text { https://orcid.org/0000-0001-6984-3979 } \\ \text { Min Young Lee } & \text { https://orcid.org/0000-0002-6860-8042 } \\ \text { Phil-Sang Chung } & \text { https://orcid.org/0000-0003-4591-2276 } \\ \text { Jae Yun Jung } & \text { https://orcid.org/0000-0002-1870-0748 }\end{array}$

\section{AUTHOR CONTRIBUTIONS}

Conceptualization: JYJ. Data curation: MTK, JHL. Formal analysis: MTK, MYL. Funding acquisition: PSC. Methodology: JHL, NTC, MYL, JYJ. Project administration: PSC, JYJ.Writing-original draft: JHL, NTC, MYL. Writing-review \& editing: all authors.

\section{SUPPLEMENTARY MATERIALS}

Supplementary materials can be found via https://doi.org/10. 21053/ceo.2019.01662.

\section{REFERENCES}

1. Hamernik RP, Patterson JH, Turrentine GA,Ahroon WA. The quantitative relation between sensory cell loss and hearing thresholds. Hear Res. 1989Apr;38(3):199-211.

2. Simonoska R, Stenberg AE, Duan M, Yakimchuk K, Fridberger A, Sahlin L, et al. Inner ear pathology and loss of hearing in estrogen receptor-beta deficient mice. J Endocrinol. 2009 Jun;201(3):397-406.

3. Caruso S, Cianci A, Grasso D, Agnello C, Galvani F, Maiolino L, et al. Auditory brainstem response in postmenopausal women treated with hormone replacement therapy: a pilot study. Menopause. 2000 May-Jun;7(3):178-83.

4. Stenberg AE, Wang H, Fish J 3rd, Schrott-Fischer A, Sahlin L, Hultcrantz M. Estrogen receptors in the normal adult and developing human inner ear and in Turner's syndrome. Hear Res. 2001 Jul;157 (1-2):87-92.

5. Nilsen J, Brinton RD. Impact of progestins on estrogen-induced neu- roprotection: synergy by progesterone and 19-norprogesterone and antagonism by medroxyprogesterone acetate. Endocrinology. 2002 Jan;143(1):205-12.

6. Moosmann B, Behl C. The antioxidant neuroprotective effects of estrogens and phenolic compounds are independent from their estrogenic properties. Proc Natl Acad Sci U S A. 1999 Aug;96(16):886772.

7. Nakamagoe M,Tabuchi K, Uemaetomari I, Nishimura B, Hara A. Estradiol protects the cochlea against gentamicin ototoxicity through inhibition of the JNK pathway. Hear Res. 2010 Mar;261(1-2):67-74.

8. Coleman JR, Campbell D, CooperWA, Welsh MG, Moyer J. Auditory brainstem responses after ovariectomy and estrogen replacement in rat. Hear Res. 1994 Nov;80(2):209-15.

9. Bittar RS, Cruz OL, Lorenzi MC, Marone SA, Miniti A. Morphological and functional study of the cochlea after administration of estrogen and progesterone in the guinea pig. IntTinnitus J. 2001;7(1):41-5.

10. Golub MS, Germann SL, Hogrefe CE. Endocrine disruption and cognitive function in adolescent female rhesus monkeys. NeurotoxicolTeratol. 2004 Nov-Dec;26(6):799-809.

11. Willott JF, VandenBosche J, Shimizu T, Ding DL, Salvi R. Effects of exposing gonadectomized and intact C57BL/6J mice to a high-frequency augmented acoustic environment: auditory brainstem response thresholds and cytocochleograms. Hear Res. 2006 Nov;221 (1-2):73-81.

12. Kim JH, Cho HT, Kim YJ. The role of estrogen in adipose tissue metabolism: insights into glucose homeostasis regulation. Endocr J. 2014;61(11):1055-67.

13. Sha SH, Taylor R, Forge A, Schacht J. Differential vulnerability of basal and apical hair cells is based on intrinsic susceptibility to free radicals. Hear Res. 2001 May;155(1-2):1-8.

14. Hultcrantz M, Sylven L, Borg E. Ear and hearing problems in 44 middle-aged women with Turner's syndrome. Hear Res. 1994 Jun; 76(1-2):127-32.

15. Wilcox SA, Saunders K, Osborn AH,Arnold A, Wunderlich J, KellyT, et al. High frequency hearing loss correlated with mutations in the GJB2 gene. Hum Genet. 2000 Apr;106(4):399-405.

16. Meltser I, Tahera Y, Simpson E, Hultcrantz M, Charitidi K, Gustafsson JA, et al. Estrogen receptor beta protects against acoustic trauma in mice: version 2. J Clin Invest. 2008 Apr;118(4):1563-70.

17. Mize AL, Shapiro RA, Dorsa DM. Estrogen receptor-mediated neuroprotection from oxidative stress requires activation of the mitogen-activated protein kinase pathway. Endocrinology. 2003 Jan;144(1):306-12.

18. Lee MY, Kabara LL, Swiderski DL, RaphaelY, Duncan RK, Kim YH. ROS scavenger, ebselen, has no preventive effect in new hearing loss model using a cholesterol-chelating agent. J Audiol Otol. 2019 Apr; 23(2):69-75.

19. Iorga A, Cunningham CM, Moazeni S, Ruffenach G, Umar S, Eghbali M. The protective role of estrogen and estrogen receptors in cardiovascular disease and the controversial use of estrogen therapy. Biol Sex Differ. 2017 Oct;8(1):33.

20. Wronski TJ, Cintron M, Doherty AL, Dann LM. Estrogen treatment prevents osteopenia and depresses bone turnover in ovariectomized rats. Endocrinology. 1988 Aug;123(2):681-6.

21. Kujawa SG, Liberman MC. Adding insult to injury: cochlear nerve degeneration after "temporary" noise-induced hearing loss: version 2. J Neurosci. 2009 Nov;29(45):14077-85. 